Original Research Paper

\title{
Correlation Based ADALINE Neural Network for Commodity Trading
}

\author{
${ }^{1}$ Chandra, J., ${ }^{2}$ M. Nachamai and ${ }^{3}$ Anitha S. Pillai \\ ${ }^{1,2}$ Department of Computer Applications, Christ University, Bangalore, Karnataka, India \\ ${ }^{3}$ Department of Computer Sciences, Hindustan University, Chennai, India
}

Article history

Received: 10-09-2015

Revised: 25-09-2015

Accepted: 20-10-2015

Corresponding Author:

Chandra, J.

Department of Computer

Science, Christ University,

Bangalore, Karnataka, India

E-mail: chandra.j@christuniversity.in

\section{Introduction}

The artificial neural network was introduced by mcculloch and Pitts and it is considered as one of the logical simulation of a biological brain. The ANN has the ability to detect future values of any unseen data object in the dataset. ANN consists of an interdependent group of artificial neurons and it processes information using a connection based approach for computation. The background knowledge is collected from its environment and is stored as interconnected neurons in the neural network. An artificial neural network is an essential method for financial services due to their ability to handle insufficient data which can process quickly. The predictive model is an important method to make variety of predictive decisions in the field of commodity trading. To determine the likelihood of the investment which is based on the current risk, the investor can decide either to sell or buy the product. Generally, all marketers look at, how consumers have behaved to the overall economy when planning on a new campaign and changes in demographics to determine if the current mix of the products will tempt the customers to make a purchase. Especially the trader who has liking for short term investment wants to make more return's on the investment will expect high optimality in the future prediction. The idea is to increase the ROI values with specific periods. So the proposed neural network model has the ability to learn by observation which is considered as one of the most powerful, feasible and very efficient method for doing predictive analytics (Arsad et al., 2012; Chandra et al., 2015b; Hall, 1999; Hammerstram, 1993; Hecht-Nielsen, 1990; Langley and Sage, 1994).

The predictive analytics is the process of extracting information from data to discover recurrent patterns. 
For any investor, the main aim of the investment made would be maximizing the values of ROI and also to reduce the investment risks. The trader will be keen on taking a decision quickly like buy or sell is based on the commodity value. Performing future prediction with respect to commodity exchange is of varying nature (Necdet and Celik, 2012; Savita et al., 2013; Sut and Şenocak, 2007; Thawornwong and Enke, 2004; Yan et al., 2006).

\section{Methodology}

There are three predictive models are suggested for an infant mortality rate. The prediction was done using Auto Regressive Integrated Moving Average (ARIMA), neural network and Linear Regression models. The performances of the prediction models are compared using Indonesia infant mortality rate data collected during 1995-2008. From the comparative study, it is found that NN model performs better than other methods and is evaluated with the help of mean absolute error, mean absolute percentage error and root mean square error (Purwanto et al., 2010). The MLP NN is used to predict the heart attack and also to diagnose cancer. The MLP NN is considered as a popular or well known method for predictive analytics (Hertz et al., 1991; Hopfield, 1982). From the literature review, it was found that the prediction models indicated similar results as far as mean square error is concerned. The MLP NN is more optimized method than other methods like self organized feature map and recurrent neural network and the performance measure is evaluated with the help of mean MMRE. The new method for stock market prediction has proposed with MLP NN and it is checked with Elmen's network (Naeini et al., 2010). It is found that the predicted values using MLP NN are very closer to the original values with higher accuracy compared to Elmen's network.

\section{Predictive Model}

The proposed predictive model is an integrated method for making effective and efficient prediction for all kinds of commodity trading. According to the investor, the integrated method works efficiently than the single method for forecasting the future values of any products in the investment. The proposed model uses Pearson's Correlation Coefficient (PCC) for feature selection and the MLP adaline NN for predicting unseen data or future data. This integrated approach works well with respect to all kinds of investment in commodity trading. Especially the current model CBFS MLP adaline feed forward NN predicts the future values of all commodity indexes. The proposed predictive model performs feature selection as the first step and then checks the efficiency of the feature selection with Pvalue summary. The selected variables are used in building neural network which user adaline neuron as a predictor. Due to the efficiency of the adaptive linear neuron, the model outperforms in prediction.

\section{Data Set Description}

The mcxindia is one of the popular web pages for the commodity trading in India. It is an ISO standard company for commodity exchange. It is a largest leading commodity futures exchange with a market share. The mcxindia data set contains more than thirteen features. The attribute of the data set are Item name, Expirymonth(dd-mm-yy), Open(Rs), High(Rs), Low(Rs), LTP(RS), PCP(RS), Change(\%), Buyqty, Sellqty, Sellprice(RS), etc. It also describes many details like mcxagri, mcxenergy and mcxmetal. The historical data is used for investigation. The data set has multiple attributes but only four attributes are considered for building the adaline NN after feature selection. The four attributes are selected using correlation values.

\section{Correlation Based Feature Selection (CBFS)}

The correlation based feature selection for machine learning in his PhD work addresses different problems associated with feature selection (Hall, 1999). The feature selection is compared with three machine learning algorithm such as C4.5, IB1 and Naïve Bayes. The correlation based feature selection is experimented with simulated data set as well as with UCI data set. The correlation based feature selection works quickly for identifying highly relevant features with better accuracy (Rubinstein and Kroese, 2004). The CBFS is a fast filter method to detect the relevant features and it is demonstrated with the real data as well as with simulated data for the experimental work which is found that effectives and efficiency of the CBFS, therefore it is proved that the method gives better efficiency and effectiveness which is considered as one of the best method for feature selection ( $\mathrm{Yu}$ and Liu, 2003; Koller and Sahaimi, 1994). The CBFS method is used to test research hypotheses when there is a linear and bi-variate relationship between any two variables. In this paper, it is found that CBFS based predictive model is used to remove highly irrelevant attributes from the data set. It is also suggested that the CBFS removes all irrelevant attributes to do any task in computational intelligence. It gives highly relevant attributes than any other methods. The predictive model makes more intellect to the investor when all the irrelevant attributes are removed for doing data analysis. The attribute subset selection is a most challenging process faced in the field of data analysis. The real world data has multiple attributes in order to reduce irrelevant attribute, the attribute subset selection method needs to be used for reducing the irrelevant data with preserved content into the whole dataset. As in data mining, all prediction models need an efficient method for doing relevant analysis. So that the good predictor methods like neural network, support vector machine may work efficiently with relevant subset. The importance of the CBFS is to improve high relevancy in the data analysis. The CBFS method can also be used for supervised and unsupervised learning 
for the selection of highly relevant features for further processing in data analysis. There are many types of correlation coefficient methods available for feature selection, only PCC was used and the highly correlated values are considered for further data analysis. The PCC method is also applied for finding non-redundant binned feature subset selection and compared with other existing methods and it found that the CBFS based feature selection works well with SVM classifier. The implementation values of CBFS using PCC values are described in Table 1.

The current research work has implemented PCC as the feature subset selection method for selecting the relevant attributes. The PCC is defined as the variance of two variables divided by the product of their standard deviation. The PCC is represented by $\mathrm{P}_{\mathrm{xy}}$, where the formula is as follows:

$$
P_{x y}=\frac{\operatorname{Cov}(x y)}{\sigma_{x} \sigma_{y}}=\sum\left[\frac{\left(x-\mu_{x}\right)\left(y-\mu_{y}\right)}{\sigma_{x} \sigma_{y}}\right]
$$

where, $\operatorname{cov}(\mathrm{xy})$ is the covariance of $\mathrm{x}$ and $\mathrm{y}, \mu_{\mathrm{x}}$ is the arithmetic mean of $\mathrm{x}, \mu_{\mathrm{y}}$ is the arithmetic mean of $\mathrm{y}$. Here the $\sigma \mathrm{x} \sigma_{\mathrm{y}}$ is the standard deviation of $\mathrm{x}$ and $\mathrm{y}$. CBFS is mainly used as an efficient and effective feature selection method in computational intelligence.

\section{Performance Measures on CBFS}

\section{P-Value Selection}

The P-values are considered as evaluation parameters to verify all associated variables. In data analysis, the Pvalue is considered as the possibility of receiving the same value for model building. If the P-value is less than or equal to the doorstep value before setting the data set, one discards the null hypothesis and accepts the trial hypothesis as applicable. For easy understanding with an impact level of ten percentages, the possibility of having the significant $\mathrm{P}$-values belongs to any one of the following criteria. The criteria for selecting the feature subset selection based on $\mathrm{P}$ value is as follows:

- If P-value lies between the values 0.01 to 0.05 then it is assumed to be very strong presumption

- If P-value belongs to 0.05 to 0.1 then it is assumed to be low presumption

- If P-value is greater than 0.1 then it is considered as no presumption. Finally, the threshold values are too optimistic when P-values reaches to less than 0.001 or 0.0053

From the above criteria it is found that, when we try to verify the relevancy of the variable which is considered to be very strong, when the P-value reaches between 0.001 to 0.0053
The P-values on PCC is implemented with the help of statistical summary in $\mathrm{R}$ tool and the measured values are described in Table 2.

\section{Neural Network Implementation}

Neural network is inspired by the computational structure found in the human brain and the neural network can imitate intelligence activities of the human brain like thinking, sensing, etc. It comprises of many processing elements called as units which are analogous to inter-neurons simply perform as intermediary processors. The MLP feed forward neural network is used for multi class classification. The Fig. 2 shows the architecture of MLP feed forward network which uses the adaptive linear regression neurons.

The process of training the MLP feed forward neural network classifier is a simple process in which the sum of weights is optimized on the basis of developing best prediction of the target variable. In order to obtain the best patterns, more computing power is needed, so to get more computing power, the current paradigm is processed quickly with in short period of time. The data set with no errors are considered for selecting both environmental and target variables. If the predicted value differs from an actual value then it considered as erroneous. The estimated accuracy of the stock market prediction using MLP feed forward NN performs better than MLP back propagation based on return on investment. Through the experimental result it is found that the MLP feed forward NN gives 95\% accuracy which higher than back propagation NN (Chandra et al., 2015a). The feed forward neural network is an artificial neural network where the connections between different units form a uni-directed cycle. In this neural network, the information moves only one direction forward from the input nodes, through the hidden nodes and to the output nodes. There are no cycles or loops in the network. The feed forward neural network uses the single layer as the hidden layer. The present model uses linear regression formula as the predictor in the $\mathrm{NN}$.

\section{Linear Regression}

The linear regression formula is used to frame the adaptive neural network for making the prediction. Linear regression is used to predict the value of a variable based on other variables (Purwanto et al., 2010). The regression analysis is used to measure the strength of the relationship between two different sets $\mathrm{X}$ and $\mathrm{Y}$. The data is modeled using linear predictor functions and the unseen variables are estimated from current variables with the linear regression function. 
Table 1. CBFS using mcxindia

\begin{tabular}{llllllllll}
\hline Buy Price.Rs & BuyQty & Change... & High.Rs. & Low. Rs. & LTP Rs & Open.Rs. & PCP. Rs. & Sell Price Rs & Sell Qty \\
\hline Buy Price.Rs. & 1 & 1 & 0 & 0 & 0 & 0 & 0 & 0 & 1 \\
Buy Qty & 1 & 1 & 1 & 1 & 1 & 1 & 1 & 1 & 1 \\
Change... & 1 & 1 & 1 & 1 & 1 & 1 & 1 & 1 & 1 \\
High.Rs. & 0 & 1 & 1 & 0 & 0 & 0 & 0 & 0 & 1 \\
Low.Rs. & 0 & 1 & 1 & 0 & 0 & 0 & 0 & 0 & 1 \\
LTP.Rs. & 0 & 1 & 1 & 0 & 0 & 0 & 0 & 0 & 1 \\
Open.Rs. & 0 & 1 & 1 & 0 & 0 & 0 & 0 & 0 & 1 \\
PCP.Rs. & 0 & 1 & 1 & 0 & 0 & 0 & 0 & 0 & 1 \\
Sell Price.Rs. & 0 & 1 & 1 & 0 & 0 & 0 & 0 & 0 & 1 \\
Sell Qty & 1 & 1 & 1 & 1 & 1 & 1 & 1 & 1 & 1 \\
\hline
\end{tabular}

Table 2. P-values on pearson's correlation coefficient using R tool

\begin{tabular}{llllllllll}
\hline Buy Price.Rs & Buy-Qty & Change & High & Low & LTP & Open & PCP & SellPrice & Sell Qty \\
\hline Sell.Qty & 0.90580 & 0.0899 & 0.39270 & 0.90500 & 0.90380 & 0.90260 & 0.90690 & 0.90530 & 0.9126 \\
Sell.Price.Rs. & $<0.0001$ & 0.6984 & 0.45800 & $<0.0001$ & $<0.0001$ & $<0.0001$ & $<0.0001$ & $<0.0001$ & 0.9126 \\
PCP.Rs. & $<0.0001$ & 0.7404 & 0.47870 & $<0.0001$ & $<0.0001$ & $<0.0001$ & $<0.0001$ & $<0.0001$ & 0.9053 \\
Open.Rs. & $<0.0001$ & 0.7416 & 0.47880 & $<0.0001$ & $<0.0001$ & $<0.0001$ & $<0.0001$ & $<0.0001$ & 0.9069 \\
LTP.Rs. & $<0.0001$ & 0.7402 & 0.48300 & $<0.0001$ & $<0.0001$ & $<0.0001$ & $<0.0001$ & $<0.0001$ & 0.9026 \\
Low.Rs. & $<0.0001$ & 0.7393 & 0.47990 & $<0.0001$ & $<0.0001$ & $<0.0001$ & $<0.0001$ & $<0.0001$ & 0.9038 \\
High.Rs. & $<0.0001$ & 0.7419 & 0.48180 & $<0.0001$ & $<0.0001$ & $<0.0001$ & $<0.0001$ & $<0.0001$ & 0.9050 \\
Change... & 0.48220 & 0.2026 & 0.48180 & 0.47990 & 0.48300 & 0.47880 & 0.47870 & 0.45800 & 0.3927 \\
Buy.Qty. & 0.74180 & 0.2026 & 0.74190 & 0.73930 & 0.74020 & 0.74160 & 0.74040 & 0.69840 & 0.0899 \\
Buy.Price.Rs. & 0.74180 & 0.4822 & $<0.0001$ & $<0.0001$ & $<0.0001$ & $<0.0001$ & $<.00010$ & $<0.0001$ & 0.9058 \\
\hline
\end{tabular}

\section{ADALINE Neural Network}

The ADALINE neural network has various inputs and one output. The adaline neural network can be used to classify objects into two different categories. The adaptive linear element minimizes the sum squared error over the training data set. However, the adaline neural network can classify objects in three manners when the objects are linearly separable (Ramasamy and Siang, 2013). The link between the input and the output neurons holds the weighted interconnections, which can change during the training phase of adaptive linear neuron.

\section{Proposed Algorithm}

The proposed algorithm describes the various steps involved in building predictive model for making the numerical prediction is shown. Using the proposed algorithm, the future trade value for all commodity tradings are predicted and becomes useful for investment.

\section{Predictive Algorithm}

1. Load the optimized data set D.

2. Perform features selection using Pearson's correlation coefficient.

3. Build neural network using adaptive linear neurons.

4.Compute $h_{1}, h_{2} \ldots . . . h_{n}$ then calculate $\log \left(h_{1}\right), \log \left(h_{2}\right) \ldots \log \left(h_{n}\right)$.

5. Perform predicted-value $=i_{n}+d_{1} h_{1+} d_{2} h_{2+} d_{n} h_{n}$.

6. Evaluate the predicted-value with the help of MRE, MMRE and then compute Accuracy Rate =1-MMRE.

7. Check If the Accuracy-rate is $>99$ then the predictedvalue is used for future prediction

Otherwise repeat step-1 to step-6 until the accuracy-rate is $>99$.

\section{Performance Measures}

The efficiency of NN method is checked with the help of MMRE and accuracy for the given data set. Using the training set to measure accuracy will typically provide an optimistically biased estimate, especially if the learning algorithm over fits the training data. Sample accuracy is an estimate of the true accuracy of the algorithm, that is, the probability of the algorithm will work correctly on classified instances drawn from the unknown distribution (Nuzzo, 2014). Generally, the error analysis is done with the help of MRE and MMRE in data analytics. In this research work, classification accuracy is the primary evaluation criteria for implementing the proposed predictive model. The classification accuracy is defined as the percentage of test data which is correctly classified by the algorithm:

$$
M R E=\frac{\mid \text { Estimated Value }- \text { Actual Value } \mid}{\text { ActualValue }}
$$

$M M R E=\frac{1}{n} *$ Sum of MRE and Accuracy $=100-$ MMRE

The MRE is an evaluation measure to check, how large as the computation value and also to standardize the measurement. The relative error is performed as the absolute error divided by the magnitude of the exact value. The absolute error is the magnitude of different variance between the actual value and the estimated value. To calculate the value of MMRE measures, the 
model uses the difference between actual and estimated value. The average differences are considered as numerical value of every observation in the data distribution and these values are considered as very sensitive to individual predictions. The predictive model is developed from a training sample where structures are randomly collected from the data set. Trade forecasting is the process of determining future values of trading or other financial trade on a commodity exchange. The successful prediction of all commodity future values could be important earnings about events whose actual outcomes have not yet been observed. The trader's might estimate some variables of interest at some specified future date. Computable forecasting models are used to forecast future data with the help of historical data. These methods are usually implemented to reduce intermediate range decisions. The adaline NN forecasts are the most costeffective methods to provide a benchmark against all other methods. Since the capabilities of such intelligence method would be difficult for an individual human mind to apprehend, the methodological singularity is seen as an occurrence beyond which events cannot be predicted.

\section{Results}

The estimated high-values are checked against the original values of the next day and then the difference between the estimated high-value and the actual high value is found as 0.566 . The same experiment is carried out randomly on another day in the same week, the difference is 0.766 . So the approximate average error rate is 0.6 for all commodity trading. Based on CBFS, only 3 variables are considered as input variables to build MLP NN. From this experimental result, it is found that the estimated accuracy is measured as 99.5 with the error rate of 0.5 . The same model can be used for making all kinds of items in commodity trading. To check the accurate value of the model, the MMR, MMRE is calculated. The total value of MRE is 0.084939 and MMRE is 0.566261 . The accuracy is measured as 99.43374 .

\section{Discussion}

Through the experimental result, it is found that the correlation based adaline MLP neural network is used as the predictor model for predicting the next high values of all commodity indexes. The short term investor who wants to buy or sell the product will benefit with this model based on the estimated high values. From this experimental work, it is also decided that the proposed predictive model can be used for making any kind of future values for different types of numeric prediction.

\section{Conclusion}

The high value of the next day commodity trading is estimated with the help of the current integrated predictive model. As an evaluation measure, the accuracy rate is calculated with the help of statistical parameter MRE, MMRE and the accuracy rate. From thelcurrent research, it is observed that the predictive high value is compared with the real-time data for the next day as final verification. The estimated error rate is very closer to zero value and the model is verified with all commodity indexes. So Correlation based adaline MLP NN can be applied for all kinds of commodity trading. The correlation based adaline MLP NN is capable of predicting exactly the same value as the next day high values for all commodities. As a further scope, the proposed predictive model can also be applied to other long term risk based investment.

\section{Acknowledgement}

The authors like to thank and acknowledge all anonymous reviewers for their suggestions to improve the quality of the paper.

\section{Author's Contributions}

Chandra, J.: Drafted and involved in experimental work.

Nachamai M: Drafted and involved in experimental work.

Anita S. Pilli: Reviewed the draft to improve the quality of the research paper.

\section{Ethics}

All materials in this paper are considered as an original work and not published in anywhere and all authors declare that there is no conflict of interest.

\section{References}

Arsad, P.M., N. Buniyamin and J.A. Manan, 2012. Neural network model to predict electrical students' academic performance. Proceeding of the International Congress on Engineering Education, Dec. 5-7, IEEE Xplore Press, Georgetown, pp: 1-5. DOI: 10.1109/ICEED.2012.6779270

Chandra, J., M. Nachamai and A.S. Pillai, 2015a. An integrated approach to predictive analytics for stock indexes and commodity trading using computational intelligence. World Comput. Sci. Inf. Technol. J.

Chandra, J., M. Nachamai, A.S. Pillai, 2015 b. Empirical estimation of multilayer perceptron for stock market indexes. ICOCOE'2015-2nd 
international Conference on Communication and Computer Engineering, Phuket, Thailand 08-62015-10-6-2015.

Hall, M.A., 1999. Correlation-based feature subset selection for machine learning. $\mathrm{PhD}$ Thesis, Department of Computer Science, University of Waikato, Waikato, N.Z.

Hammerstram, D., 1993. Working with neural network. IEEE Spectrum.

Hecht-Nielsen, R., 1990. Neurocomputing. 3rd Edn., Addison-Wesley Publishing Company, Reading, Mass, ISBN-10: 0201093553, pp: 433.

Hertz, J., A. Krogh and R. Palmer, 1991. Introduction to the Theory of Neural Computation. 1st Edn., Addison-Wesley Pub. Co., Redwood City, ISBN10:0201503956, pp: 327.

Hopfield, J., 1982. Neural networks and physical systems with emergent collective computational abilities. Proc. Natil. Acad. Sci. U.S.A., 79: 2554-2558. PMID: 6953413

Koller, D. and M. Sahaimi, 1994. Towards optimal feature selection. Proceedings of the $3 \mathrm{rd}$ International Conference on Machine Learning, (ICM' 94), pp: 284-292.

Langley, P. and S. Sage, 1994. Induction of selective bayesian classifier. Proceedings of 10th International Conference on Uncertainty in Artificial Intelligence, (ICU' 94), San Francisco, pp: 399-406.

Naeini, M.P., H. Taremian and H.B. Hashemi, 2010. Stock market value prediction using neural networks. Proceedings of the International Conference on Computer Information Systems and Industrial Management Applications, Oct. 8-10, IEEE Xplore Press, Krackow, pp: 132-136.

DOI: 10.1109/CISIM.2010.5643675

Necdet, S. and Y. Celik, 2012. Prediction of mortality in stroke patients using multilayer perceptron neural networks. Turk J. Med. Sci., 42: 886-893.

Nuzzo, R., 2014. Scientific method: Statistical errors. Nature, 506: 150-150. DOI: 10.1038/506150

Purwanto, C. Eswaran and R. Logeswaran, 2010. A comparison of ARIMA, neural network and linear regression models for the prediction of infant mortality rate. Proceedings of the 4th Asia International Conference on Mathematical/Analytical Modelling and Computer Simulation, May 26-28, IEEE Xplore Press, Kota Kinabalu, pp: 34-39. DOI: 10.1109/AMS.2010.20

Rubinstein, R.Y. and D.P. Kroese, 2004. Convergence of actual and predicted share prices-an adaline neural network approach. Global J. Comput. Sci. Technol. Network, Web Security.

Ramasamy, R. and T.C. Siang, 2013. Convergence of actual and predicted share prices-an adaline neural network approach. Global J. Comput. Sci. Technol. Network, Web Security.
Savita, D.S. Kapoor and A.K. Kohli, 2013. Comparative evaluation of generalized ADALINE using variable learning rate parameter. Int. J. Computational Eng. Management, 16: 2230-7893.

Sut, N. and M. Şenocak, 2007. Assessment of the performances of multilayer perceptron neural networks in comparison with recurrent neural networks and two statistical methods for diagnosing coronary artery disease. Expert Syst., 24: 131-42.

Thawornwong, S. and D. Enke, 2004. Forecasting stock returns with artificial neural network. G.P. IRM Press.

Yan, H., Y. Jiang, J. Zheng, C. Peng and Q. Li, 2006. A multilayer perceptron-based medical decision support system for heart disease diagnosis. Expert Syst. Applic., 30: 272-81.

DOI: $10.1016 /$ j.eswa.2005.07.022

$\mathrm{Yu}, \mathrm{L}$. and H. Liu, 2003. Feature selection for highdimensional data: A fast correlation-based filter solution. Proceedings of the 12th International, D.C., Conference on Machine Learning (ICML' 03), Washington pp: 856-863.

Table 1 shows the pair wise correlation coefficient values using statistical summary in $\mathrm{R}$ tool. To select the relevant attributes, the highly correlated variables are considered for doing further analysis. To determine the variable as highly correlated, the correlated values which are near to one or equal to one is considered as the highly associated variables or related variables. As an average, any correlated value which is very closer to one is considered as highly correlated variables. From table 1 the attributes 'open', 'low', 'high-values' and 'sellingprice' are considered as highly correlated variables. The correlated variables are verified with the help of statistical parameter P-value as a performance measure, so the $\mathrm{P}$-value calculation is considered as an evaluation parameter for verifying the correlated variables.

From Table 2, the P-value measures are shown, based on the criteria for selecting the variables, whichever variables scored between 0.01 to 0.005 are confirmed as highly correlated, which are fed as inputs for building adaptive linear regression NN.

Figure 4 and Table 3 describes the experimental result of predictor variable with help of all hidden variables. Once the high-values are estimated, the predictive high values are calculated. The predictive parameters for checking the accuracy of the high-values are performed with the help of the variables Error, MRE, MMRE and Accuracy. The MLP feed forward adaline NN is implemented using NNET package in R tool.

Figure 1 describes various methods involved in predictive analysis Model which contains the data load from popular web pages like MCX and NSE data set. Once the data is selected, CBFS is used as an efficient feature selection for relevant analysis. The relevant features are used build the proposed model for the future prediction. The Adaline NN is used as a predictor for the next 'High- value' prediction. 
Chandra J. et al. / Journal of Computer Science 2015, 11 (7): 863.871 DOI: $10.3844 /$ jessp.2015.863.871

Table 3. Experimental result for high-value prediction

\begin{tabular}{llllll}
\hline Item no & Open & High & Predictive high-value & Error & MRE \\
\hline 1 & 116 & 116.2 & 116.3515 & 0.001538 & $1.32 \mathrm{E}-05$ \\
2 & 114.8 & 115.5 & 114.7678 & 0.282232 & 0.002453 \\
3 & 113.8 & 114.9 & 113.9162 & 0.383806 & 0.003358 \\
4 & 112.6 & 113.6 & 112.4158 & 0.534212 & 0.00473 \\
5 & 115.4 & 115.4 & 115.1856 & 0.064383 & 0.000559 \\
6 & 113.9 & 114.8 & 113.887 & 0.413033 & 0.003614 \\
7 & 112.6 & 113.5 & 112.5893 & 0.360658 & 0.003193 \\
8 & 985 & 990 & 977.7658 & 5.234239 & 0.005325 \\
9 & 970 & 962.1713 & 5.028662 & 0.005199 \\
10 & 964 & 948.5 & 936.0378 & 6.962206 & 0.007383 \\
11 & 937.4 & 922 & 907.6443 & 9.555696 & 0.010418 \\
12 & 910 & 957.5 & 928.0873 & 13.91272 & 0.014769 \\
13 & 955 & 416.2 & 414.8202 & 2.029763 & 0.004869 \\
14 & 415.4 & 408.9373 & 3.562713 & 0.008637 \\
15 & 409.2 & 404.9365 & 4.263489 & 0.010419 \\
& 406.2 & 409.6 & & & Total= \\
& & & & & 0.084939 \\
& & & & & MMRE = \\
& & & & & 0.566261 \\
& & & & Accuracy = \\
& & & & 99.43374 \\
\hline
\end{tabular}

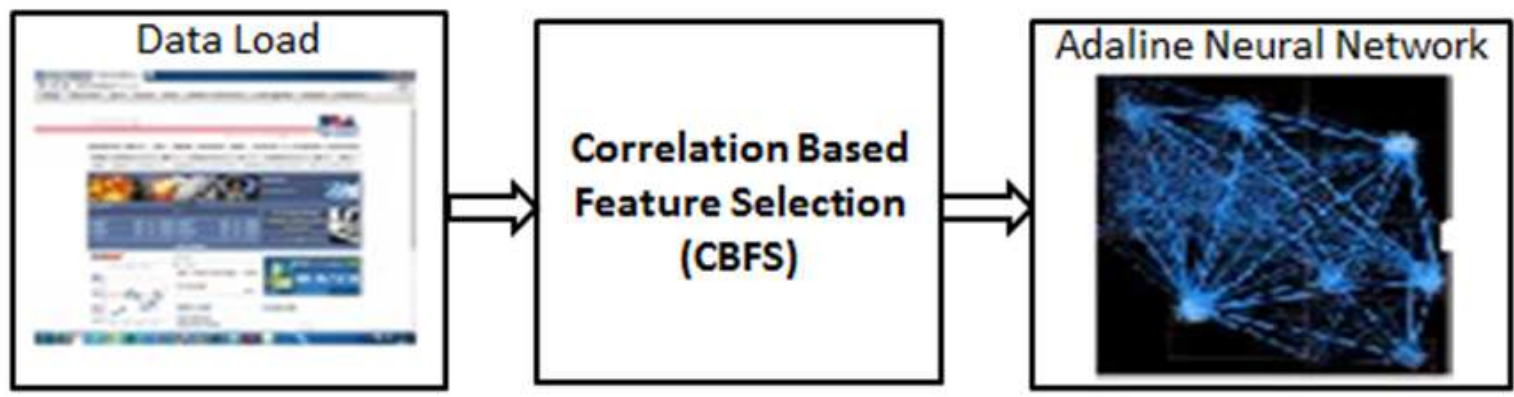

Fig. 1. Block diagram for proposed model

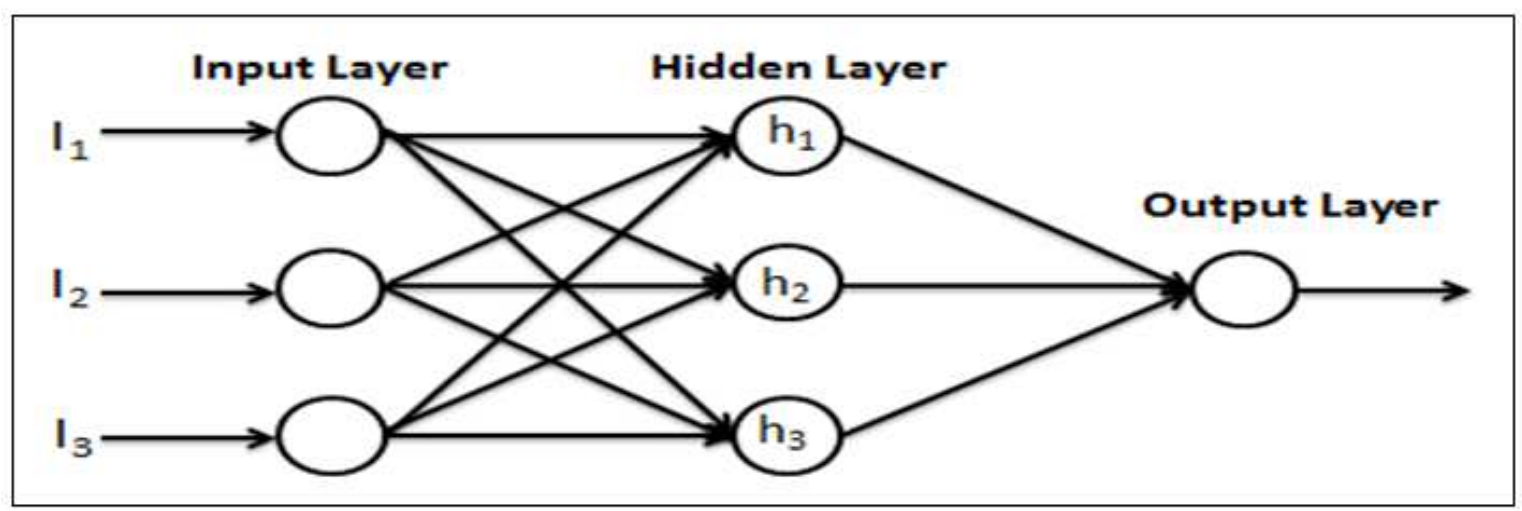

Fig. 2. MLP feed forward neural network architecture 


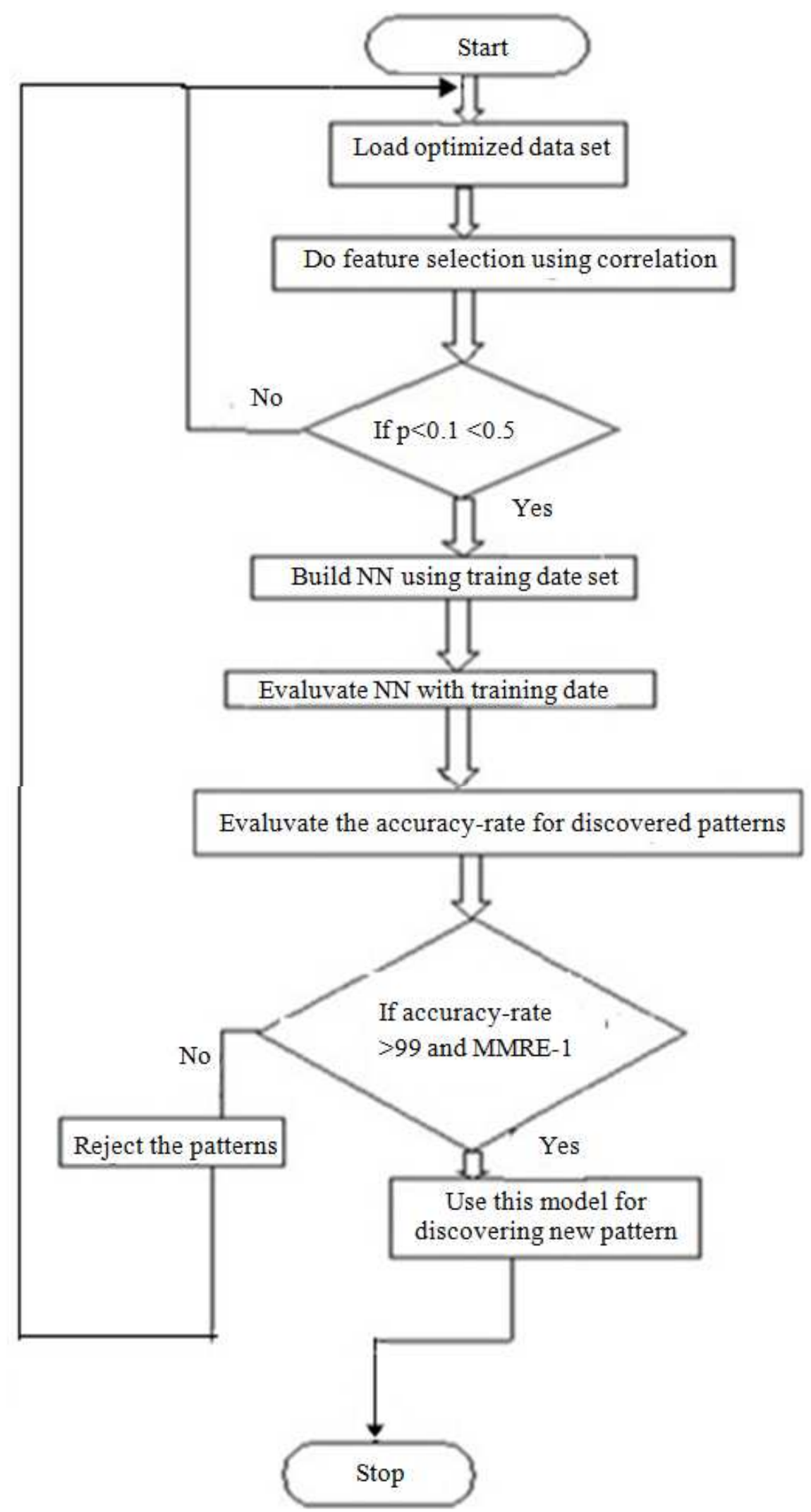

Fig. 3. Proposed frameworks for building predictive model 
Chandra J. et al. / Journal of Computer Science 2015, 11 (7): 863.871

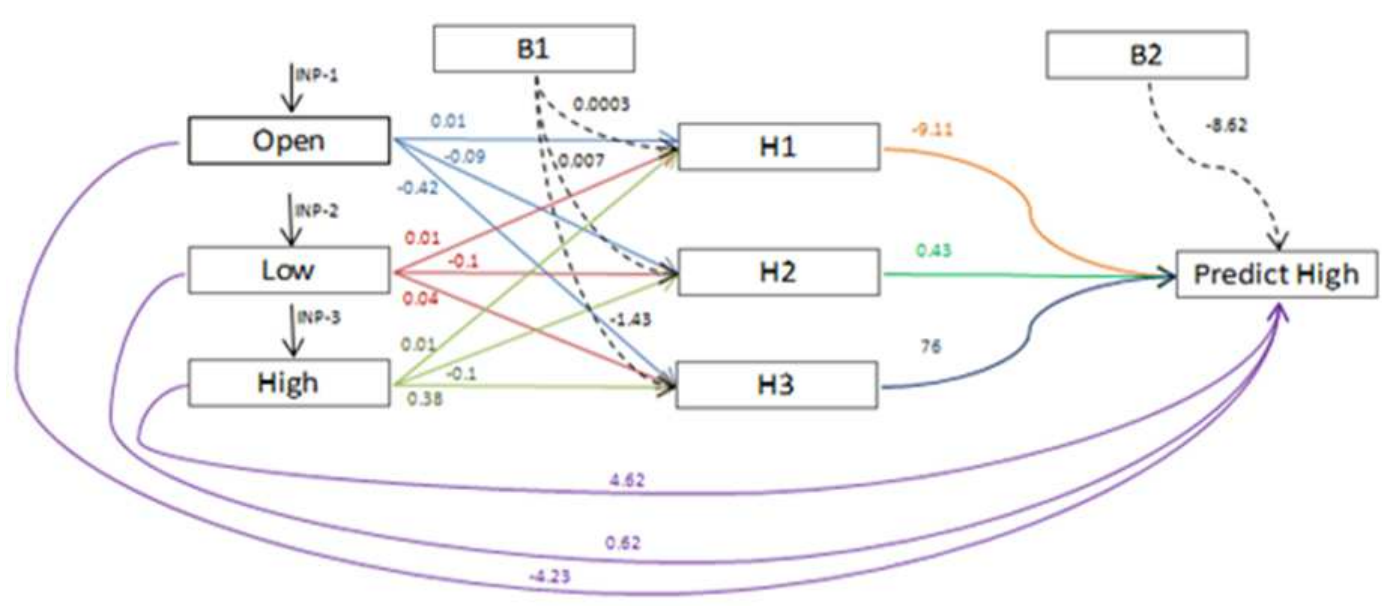

Fig. 4. MLP feed forward adaptive NN 